\title{
In Electrospray Ionization Source Hydrogen/ Deuterium Exchange LC-MS and LC-MS/MS for Characterization of Metabolites
}

\author{
Wing Lam and Ragu Ramanathan \\ Department of Pharmacokinetics, Dynamics, and Metabolism, Pfizer Global Research and Development, \\ Ann Arbor, Michigan, USA
}

\begin{abstract}
A new method is described for performing hydrogen/deuterium (H/D) exchange in an electrospray ionization (ESI) source. The use of liquid chromatography (LC)-mass spectrometer equipped with an ESI source and deuterium oxide $\left(\mathrm{D}_{2} \mathrm{O}\right)$ as the sheath liquid allows $\mathrm{H} / \mathrm{D}$ exchange experiments to be performed on-line. This directly provides information for determining the number and position of exchangeable hydrogens, aiding in the elucidation of the structures of drug metabolites. To demonstrate the utility of this method, LC-mass spectrometry (MS) and LC-MS/MS experiments were performed using either $\mathrm{H}_{2} \mathrm{O}$ or $\mathrm{D}_{2} \mathrm{O}$ as sheath liquid on a matrix metalloprotease (MMP) inhibitor (PD 0200126) and its metabolites. Examination of the mass shift of the deuteriated molecule from that of the protonated molecule allowed the number of exchangeable protons to be determined. Interpretation of the production-spectra helped to determine the location of the exchanged protons and assisted in the assignment of the site(s) of modification for each metabolite. (J Am Soc Mass Spectrom 2002, 13, 345-353) (C) 2002 American Society for Mass Spectrometry
\end{abstract}

$\mathrm{D}$ uring the process of drug discovery, it is highly desirable to increase the number of successful drug candidates for preclinical, clinical and commercial development. Therefore, the drug discovery process is constantly scrutinized and improved [1]. Adding to this pressure is the generation of vast numbers of new chemical entities resulting from combinatorial chemistry technology [2]. Drug metabolism plays an important role in the drug discovery process [3]. Specifically, the identification of metabolites during the early stage of development can be helpful to medicinal chemists trying to block some of the metabolic hot spots and produce an appropriate drug that is less susceptible to metabolism and increase the half-life of the drug. Therefore, rapid identification of drug metabolites is imperative for drug development [4,5].

Hydrogen/deuterium exchange is a well-established technique for studying structure, stability, folding dynamics, and intermolecular interactions in proteins in solution [6]. During solution phase H/D exchange, labile protons in the side chains and amide hydrogens, which are not protected from solution generally exchange rapidly. Exchanges of these unprotected hydrogens occur on the order of a few to a few tenths per second under the experimental conditions described in

Published online February 13, 2002

Address reprint requests to Dr. R. Ramanathan, Department of Pharmacokinetics, Dynamics, and Metabolism, Pfizer Global Research and Development, 2800 Plymouth Road, Ann Arbor, MI 48105. E-mail: ragu. ramanathan@pfizer.com the aforementioned studies. If however, amide or side chain hydrogens are protected from solution (e.g., when they are hydrogen-bonded in structurally stable secondary-structure elements), the exchange rates can be considerably reduced. Methods in which H/D exchange experiments are combined with either nuclear magnetic resonance (NMR) spectroscopy or mass spectrometry are also well-established [7, 8]. NMR methods, when coupled with H/D exchange are the ideal choice for monitoring individual residues or each amide hydrogen; however, these methods are limited to highly purified proteins or metabolites that are soluble at high concentrations, thus eliminating the possibility of determining structural features of drugs and metabolites that are in the discovery stage of drug development and are only available in limited quantities. On the contrary, mass spectrometry methods have advantages from inherent sensitivity and selectivity.

As early as 1971, Hunt et al. [9] showed that under chemical ionization (CI) conditions, H/D exchange can be rapidly achieved by using either $\mathrm{CH}_{3} \mathrm{OD}$ or $\mathrm{ND}_{3}$ as reagent gas. Later, Hunt and Sethi [10] showed that the proton affinity difference between the analyte and the reagent gas is a major factor in determining the degree of exchange in chemical ionization mass spectrometry. Isotopic exchange studies performed in a collision cell of a tandem mass spectrometer was used to show that $\mathrm{CH}_{3} \mathrm{OD}$ exchanged specific acidic hydrogens of phenols and carboxylic acids but not the amino hydrogens [11]. 
Gas-phase H/D exchange kinetics of several protonated amino acids and dipeptides have also been reported [12]. Although limited, H/D exchange reactions have been used in the structural studies in combination with fast atom bombardment (FAB) [13], Frit-FAB [14], and thermospray [15, 16] ionization techniques.

Hydrogen/deuterium exchange studies involving ESI produced ions are relatively new. Its feasibility was originally demonstrated by Katta and Chait [17, 18] when they showed that charge state distribution of proteins in ESI mass spectra, to some extent, reflect the amount of protonation of proteins in solution and ESI spectra can be used to study conformational changes of proteins in solution. Later, this technique was extended to quality control studies involving recombinant protein drugs [19], chaperonian protein folding [20], ligand-binding studies [21], and for screening combinatorial libraries of de novo proteins [22]. In addition, $\mathrm{H} / \mathrm{D}$ exchange methods have also been expanded to study conformational differences of multiply protonated gas-phase proteins [23].

Aforementioned methods, however, are not suitable for accommodating the needs of the pharmaceutical industry because during the drug discovery process, rapid and sensitive on-line methods are required for identification of the metabolites. In an attempt to achieve this, Hemling et al. [24] used $\mathrm{ND}_{3}$ as the nebulizer or curtain gas and showed the feasibility of H/D exchange for structure elucidation of ESI formed ions. Demonstration of this method, however, was limited to infusion of purified sample into a mass spectrometer. Furthermore, the usefulness of this method for obtaining tandem mass spectrometry information was not demonstrated. Recently, Liu et al. [25] and Ohashi et al. [26] used deuterium oxide as the LC mobile phase to enable on-line H/D exchange experiments to facilitate drug metabolite identification. This technique, however, required preparation of all the samples in $\mathrm{D}_{2} \mathrm{O}$ and using all deuteriated buffer and mobile phase which in turn lacks the practical utility for rapid metabolite identification. To date, a practical ESI based on-line H/D exchange or similar method for characterization of drug metabolites remain unknown.

In this paper, we describe our experimental effort in achieving on-line type H/D exchange for characterization of metabolites of a matrix metalloprotease (MMP) inhibitor (PD 0200126). MMP are a class of zinc containing enzymes that are responsible for the degradation of extracellular matrix components such as collagen. Although the role of MMP is not completely understood, they are implicated in a wide range of vascular diseases, including inflammation, smooth muscle cell migration, and the pathogenesis of vascular lesions [27]. LCESI-MS and LC-ESI-MS/MS spectra obtained on an ion trap mass spectrometer using either $\mathrm{H}_{2} \mathrm{O}$ or $\mathrm{D}_{2} \mathrm{O}$ as sheath liquid were compared to aid in identification of the metabolites of PD 0200126.

\section{Experimental}

$\left[{ }^{14} \mathrm{C}\right] \mathrm{PD}$ 0200126， 2-(4'-bromo-biphenyl-4-sulfonylami no)-N-hydroxy-3-methyl-butyramide [27] and the proposed metabolites $\mathrm{M}_{8}$ (PD 0408208), $\mathrm{M}_{9}$ (PD 0169469), and $\mathrm{M}_{10}$ (PD 0307442) were synthesized at Pfizer Global Research \& Development (Ann Arbor, MI) and confirmed by NMR, LC-MS, and LC-MS/MS [27].

\section{In Vivo Study}

Blood collected at various time points following a 300 $\mathrm{mg} / \mathrm{kg}$ oral administration of PD 0200126 to rabbits was used to obtain plasma samples. Plasma was precipitated by addition of three volumes of acetonitrile to one volume of plasma and then centrifuging and transferring the supernatant and drying it down. The dried sample was reconstituted in mobile phase and a 100-uL aliquot was injected for each experiment. Details of the in vivo experiment are described elsewhere [28].

\section{In Vitro Study: HPLC and MS Conditions}

The proposed metabolite $\mathrm{M}_{2}$ (PD 0200126-glucuronide) was formed following incubation of $\left[{ }^{14} \mathrm{C}\right] \mathrm{PD} 0200126$ with dog liver hepatocytes for $180 \mathrm{~min}$ and the incubation conditions have been described [28].

HPLC grade solvents were purchased from Mallinkrodt Baker, Inc. (Paris, KY). Water was purified using the Millipore Milli- $Q_{\text {plus }}$ water purification system (Bedford, MA). Ultima Flo M scintillation cocktail was purchased from Packard Instrument Co. (Meridan, CT). A $2.0 \mathrm{ng} / \mu \mathrm{L}$ stock solution of PD 0200126 was prepared in methanol, and $200 \mathrm{ng}$ of each analyte standard was injected for analysis.

The HPLC system consisted of Agilent 1100 (Agilent Technologies, Wilmington, DE) pumps with an Agilent 1100 autoinjector and a UV detector. Separation of metabolites was achieved using a Metasil AQ guard column (Metachem Technologies, Torrance, CA) and a $150 \times 4.6 \mathrm{~mm}$ MetaChem Polaris C-18 reversed-phase HPLC column (Metachem Technologies). The mobile phase, which consisted of $20 \mathrm{mM}$ ammonium acetate in water adjusted to $\mathrm{pH} 4$ with acetic acid (Solvent $\mathrm{A}$ ) and acetonitrile (Solvent $\mathrm{B}$ ), was maintained at a constant flow rate $(1 \mathrm{~mL} / \mathrm{min})$. Gradient elution of metabolites was achieved using programmed stepwise changes of $\mathrm{A} / \mathrm{B}$ ratios starting at $95 / 5,50 / 50,25 / 75,5 / 95$, and $95 / 5$ at $0,40,50,60$, and $75 \mathrm{~min}$, respectively. After passing through the UV detector $(280 \mathrm{~nm})$, the HPLC effluent was split so that $20-30 \%$ of the flow was introduced into the mass spectrometer via the supplied ESI source while $70-80 \%$ was diverted to the radioactivity detector. The analog output from the UV and radioactivity detector, along with the MS signal were recorded in real time by the data system provided with the mass spectrometer (Xcalibur V 1.2, Thermo Finnigan). A separate software program (Win-Flow V 1.5, IN/US, Tampa, FL) was used to control the $\beta$-RAM and 


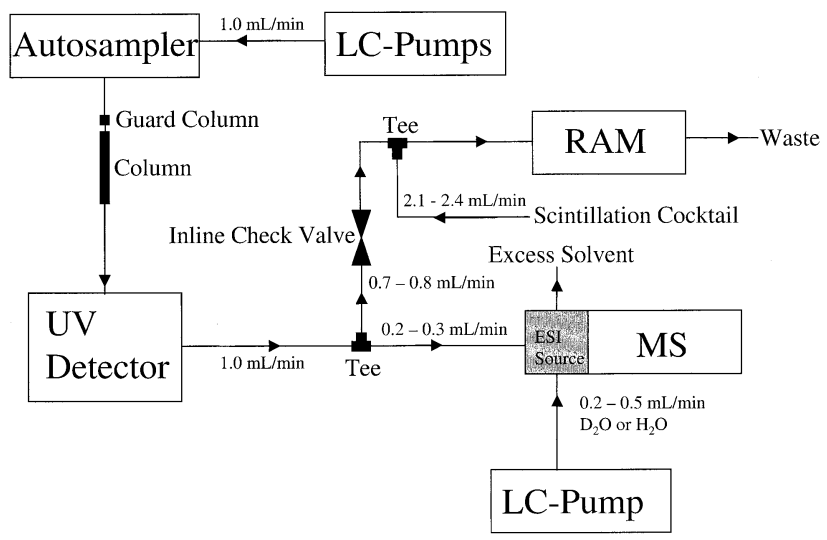

Figure 1. Schematic showing the instrumental setup used for the in ESI source H/D exchange.

also collect the analog output from the $\beta$-RAM and the $\beta$-RAM data was integrated to obtain semi-quantitative information about the metabolites and the unchanged drug. The delay in response between the radioactivity detector and MS was 0.2-0.4 min with the MS response being recorded first.

Sheath liquid, either $\mathrm{H}_{2} \mathrm{O}$ or $\mathrm{D}_{2} \mathrm{O}$, was delivered at a constant flow rate of 0.2 or $0.4 \mathrm{~mL} / \mathrm{min}$ by a PE Series 200 pump (Perkin Elmer, Norwalk, CT) (Figure 1). LC-MS (scan range 100-1100 Da) and LC-MS/MS experiments were performed using an ion trap (LCQDeca, Thermo Finnigan, San Jose, CA) mass spectrometer equipped with an ESI ion source. Operating conditions for ESI in the negative ionization mode were as follows: spray voltage, $4500 \mathrm{~V}$; capillary temperature, $300{ }^{\circ} \mathrm{C}$; sheath gas $\left(\mathrm{N}_{2}\right), 80$ (arbitrary units); and auxiliary gas $\left(\mathrm{N}_{2}\right), 20$ (arbitrary units). For all LC-MS/MS experiments performed using the ion trap mass spectrometer, helium was maintained at a constant pressure of 40 psi and used as the damping gas as well as the collision gas. For all LC-MS ${ }^{n}$ experiments, the precursor isolation window was set at 1 Thompson and the activation amplitude (\%), activation $\mathrm{Q}$, and activation time (ms) were set at 35, 0.25, and 30, respectively. Generally, 10-15 scans were signal averaged to obtain a final MS or MS/MS spectrum.

\section{Results and Discussion}

The structures of all the drug candidates and the metabolites studied as well as the maximum number of exchangeable hydrogens associated with each of the chemical entities are listed in Table 1. Structure elucidation of these metabolites was achieved by full scan LC-MS and MS/MS with and without hydrogen/deuterium exchange. Under the LC-MS conditions used, PD 0200126 eluted at $38.6 \mathrm{~min}$ and deprotonated molecules $\left([\mathrm{M}-\mathrm{H}]^{-}\right)$containing ${ }^{79} \mathrm{Br}$ and ${ }^{81} \mathrm{Br}$ were detected at $m / z 425$ and 427, respectively (Figure 2 bottom panel). When $\mathrm{D}_{2} \mathrm{O}$ was used as the sheath liquid at a flow rate of $0.2 \mathrm{~mL} / \mathrm{min}$, the most abundant isotopic envelope was observed to shift by 2 Da to $m / z 427$ and
429 (Figure 2 middle panel). The most intense peak in the mass spectrum, $m / z 427$, was associated with replacement of the maximum number of exchangeable protons (i.e., hydrogens on heteroatoms such as $\mathrm{O}, \mathrm{N}$, and $S$ ) and associated removal of a deuterium for ionization. Although ions of $\mathrm{m} / \mathrm{z} 427$ and 429 are abundant among the isotopic cluster ions observed, detection of ions of $\mathrm{m} / \mathrm{z} 426$ and 428 indicated that the exchange has not been driven to completion. Absence or presence of negligible amounts of ions of $\mathrm{m} / \mathrm{z} 425$ following the H/D exchange (Figure 2 middle panel) suggests that PD 0200126 is undergoing exchange to replace at least two of the three active hydrogens. As explained by Palmer et al. [29] during a similar H/D exchange experiment involving CE-MS, incomplete exchange can be associated with either the rate of the exchange or back-exchange. In other words, if the exchangeable hydrogens are not equally active, only the most active hydrogen will undergo the exchange. Thus, making the process rate limiting. On the other hand, back-exchange can also contribute to incomplete exchange because of the presence of $\mathrm{H}_{2} \mathrm{O}$ in solvents or inadequate amounts of $\mathrm{D}_{2} \mathrm{O}$.

To explore these two factors associated with incomplete hydrogen/deuterium exchange and how they influence structural elucidation, we undertook systematic evaluation of the sheath liquid H/D exchange process. Our first evaluation was around the sheath liquid flow rate. Increasing the sheath liquid flow rate from 0.2 to $0.4 \mathrm{~mL} / \mathrm{min}$ increased the theoretical atom $\%$ of deuterium in the ESI source from $62 \%(0.2 /(0.125$ +0.2 ), where 0.125 is the estimated flow of aqueous buffer entering the source at $\sim 40 \mathrm{~min}$ and 0.2 is the $\mathrm{D}_{2} \mathrm{O}$ sheath liquid flow rate) to $76 \%(0.4 /(0.125+0.4))$ [30] and resulted in limited change on the isotopic cluster pattern. However, reducing the sheath liquid flow rate to $0.1 \mathrm{~mL} / \mathrm{min}$ completely shifted the isotopic cluster pattern and gave a nearly Gaussian distribution of peaks. To confirm the experimental \% D incorporation and to compare with the theoretical values of $62-76 \%$ and to calculate the number exchangeable hydrogens, we used the step-by-step method described by McCloskey et al. [30, 31]. The mass spectrum of PD 0200126 obtained before exchange (Figure 2) showed $m / z 425$, $426,427,428$, and 429 peaks to have relative intensities (\%) of 39.3, 8.2, 41.1, 8.5, and 2.9, respectively. Following in-ion-source exchange, the isotopic cluster ions of $\mathrm{m} / \mathrm{z}$ $425,426,427,428,429,430$, and 431 were detected with respective relative intensities (\%) of 1.1, 10.7, 33.3, 14.6, 32.8, 5.9, and 1.6. Our calculations using the method described by McCloskey and co-workers [30, 31] showed that PD 0200126 underwent two exchanges and the experimentally determined \% D incorporation to be less than $50 \%$. Although the calculations were complex due to the presence of multiisotopic elements, results show that in-source H/D exchange is most likely limited by the $\%$ of deuterium available for the exchange and rate limited.

To further assess the effect of exchange rate and back 
Table 1. List of metabolites, the corresponding number of exchangeable protons associated with each metabolite, deprotonated and dedeuteriated ion cluster information

\begin{tabular}{|c|c|c|c|c|}
\hline Drug/Metabolite & Structure & Max-Ex & {$[\mathrm{M}-\mathrm{H}]^{-}$} & $*[\mathrm{M}-\mathrm{D}]$ \\
\hline PD 0200126 & & 3 & $425 / 427$ & $427 / 429$ \\
\hline $\begin{array}{l}\text { PD 0200126- } \\
\text { glucuronide }\left(\mathrm{M}_{2}\right)\end{array}$ & & 6 & $601 / 603$ & $606 / 608$ \\
\hline $\begin{array}{l}\text { Monohydroxy- } \\
\text { PD } 0307442\left(\mathrm{M}_{7}\right)\end{array}$ & & 4 & $425 / 427$ & $428 / 430$ \\
\hline PD $0408208\left(\mathrm{M}_{8}\right)$ & & 2 & $310 / 312$ & $311 / 313$ \\
\hline PD $0169469\left(\mathrm{M}_{9}\right)$ & & 2 & $410 / 412$ & $411 / 413$ \\
\hline PD $0307442\left(\mathrm{M}_{10}\right)$ & & 3 & $409 / 411$ & $411 / 413$ \\
\hline
\end{tabular}

Max-Ex: Maximum Number of Exchangeable Protons

*Highest molecular ion pair of the molecular ion cluster. exchange, we incubated $200 \mu \mathrm{L}$ of the PD 0200126 standard with $800 \mu \mathrm{L}$ of $\mathrm{D}_{2} \mathrm{O}$ for $24 \mathrm{~h}$ and used deuteriated mobile phase along with $\mathrm{D}_{2} \mathrm{O}$ sheath liquid and repeated the LC-MS experiment. Isotopic cluster

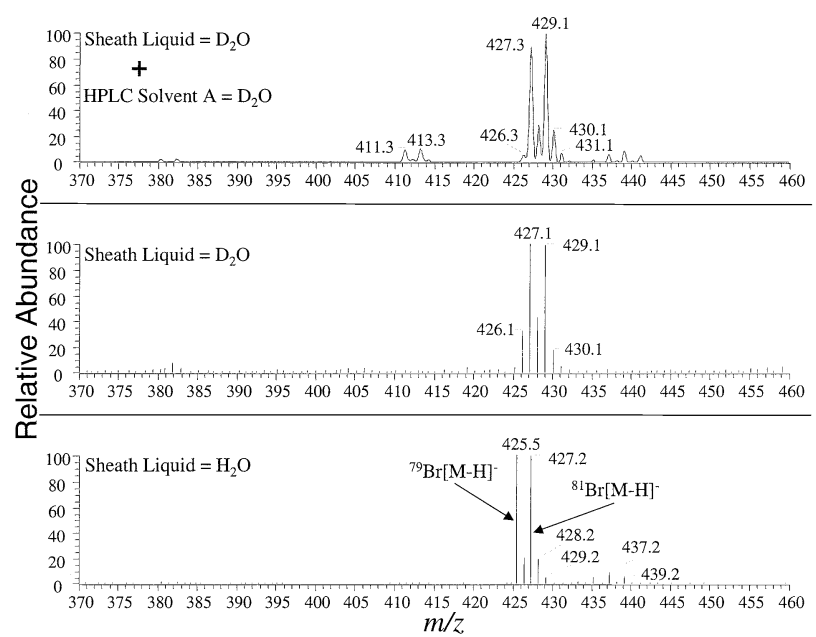

Figure 2. A comparison of LC-MS spectra of PD 0200126 standard obtained using off-line H/D exchange followed by HPLC using deuteriated mobile phase and $\mathrm{D}_{2} \mathrm{O}$ as the sheath liquid (top panel), on-line $\mathrm{H} / \mathrm{D}$ exchange using aqueous mobile phase and $\mathrm{D}_{2} \mathrm{O}$ as the sheath liquid (middle panel), and aqueous mobile phase and $\mathrm{H}_{2} \mathrm{O}$ as the sheath liquid (bottom panel). pattern (Figure 2 top panel) with less abundant ions of $\mathrm{m} / \mathrm{z} 426$ indicated that the exchange has been driven to near completion compared to previous experiments where the H/D exchange was limited to occur only in the ion source region. Additionally, this experiment confirmed that in-source sheath liquid $\mathrm{H} / \mathrm{D}$ exchange is less efficient than off-line solvent exchange. However, compared to previously described on-line type H/D exchange methods $[25,26]$ using in-source $H / D$ exchange, information about the exchangeable hydrogens of the metabolites can be readily made available.

Next, we evaluated the use of in-ion-source H/D exchange for structure elucidation of the metabolites. Initial experiments were performed using mobile phase system (HPLC flow rate of $1 \mathrm{~mL} / \mathrm{min}$ ) consisted of 20 $\mathrm{mM}$ ammonium acetate in water adjusted to $\mathrm{pH} 4$ with acetic acid (Solvent A) and acetonitrile (Solvent $\mathrm{B}$ ) and the sheath liquid $(0.2 \mathrm{~mL} / \mathrm{min})$ was either $\mathrm{H}_{2} \mathrm{O}$ or $\mathrm{D}_{2} \mathrm{O}$. This set of experiments limited the hydrogen/deuterium exchange to take place only in the ion source region. A comparison of the product-ion-spectra obtained using $\mathrm{H}_{2} \mathrm{O}$ and $\mathrm{D}_{2} \mathrm{O}$ as sheath liquid is shown (Figure 3 bottom and middle panels). The next set of experiments was performed using mobile phase system (HPLC flow rate of $1 \mathrm{~mL} / \mathrm{min}$ ) consisting of $20 \mathrm{mM}$ ammonium acetate in $\mathrm{H}_{2} \mathrm{O}$ or $\mathrm{D}_{2} \mathrm{O}$ adjusted to $\mathrm{pH} 4$ 


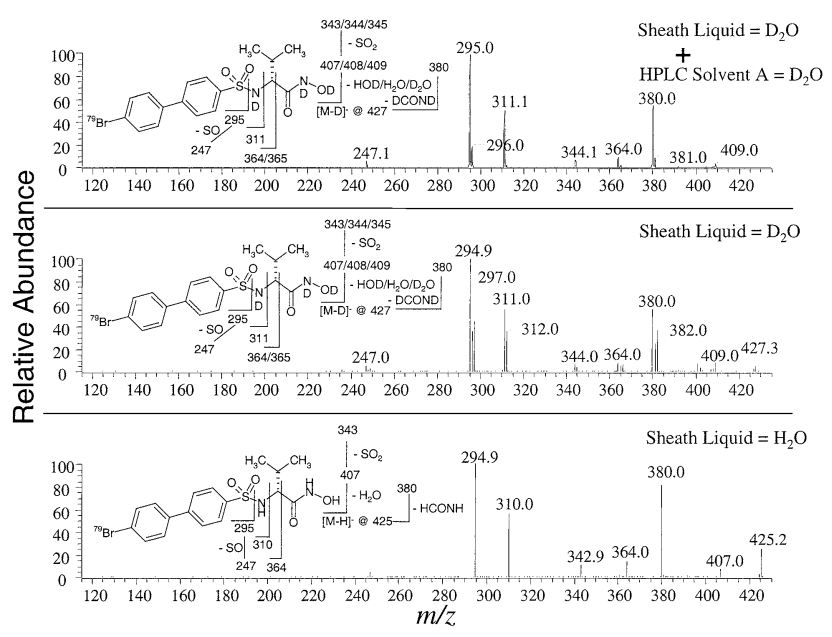

Figure 3. A comparison of LC-MS/MS spectra of PD 0200126 standard (MS isolation of deprotonated molecules that contained ${ }^{79} \mathrm{Br}$ ) obtained using off-line $\mathrm{H} / \mathrm{D}$ exchange followed by HPLC using deuteriated mobile phase and $\mathrm{D}_{2} \mathrm{O}$ as the sheath liquid (top panel), on-line $\mathrm{H} / \mathrm{D}$ exchange using aqueous mobile phase and $\mathrm{D}_{2} \mathrm{O}$ as the sheath liquid (middle panel), and aqueous mobile phase and $\mathrm{H}_{2} \mathrm{O}$ as the sheath liquid (bottom panel).

with acetic acid (Solvent A) and acetonitrile (Solvent B) and respectively $\mathrm{H}_{2} \mathrm{O}$ or $\mathrm{D}_{2} \mathrm{O}$ as the sheath liquid at a flow rate of $0.2 \mathrm{~mL} / \mathrm{min}$. This set of experiments allowed the hydrogen/deuterium exchange to occur before injection on the column and increased the \% D incorporation by the use of $\mathrm{D}_{2} \mathrm{O}$ as the mobile phase (Figure 3 top panel). Comparison of the middle and top panels of Figure 3 clearly shows that H/D exchange has not been driven to completion in the in ESI ion source $\mathrm{H} / \mathrm{D}$ exchange process. Presence of fragment ions of $\mathrm{m} / \mathrm{z} 249,297,312$, and 382 indicates that some of the precursor ions of $\mathrm{m} / \mathrm{z} 427$ that got isolated for MS/MS are from ${ }^{81} \mathrm{Br}$-PD 0200126 and the presence of abundant ions of $m / z 247,295,311$, and 380 indicates that most of the $\mathrm{m} / \mathrm{z} 427$ that got selected for MS/MS are from ${ }^{79} \mathrm{Br}$-PD 0200126 that had undergone three deuterium exchanges (net gain of two due to removal of an exchangeable proton for ionization). The shift of the fragment ion of $\mathrm{m} / \mathrm{z} 310$ (Figure 3 bottom panel) to $\mathrm{m} / \mathrm{z}$ 311 (Figure 3 middle and top panels) clearly shows that the amide nitrogen on the bromo-biphenyl-sulfonamide moiety is intact. Absence of the fragment ions of $\mathrm{m} / \mathrm{z}$ 249, 297, 312, and 382 in the MS/MS spectrum obtained following off-line H/D exchange for $24 \mathrm{~h}$ (Figure 3 top panel) clearly indicates that H/D exchange has been driven to completion and under the experimental conditions used \% D available was much higher than $62-76 \%$ deuterium made available for the in-ion- source exchange experiments. In addition, the back exchange was limited or absent in solvent exchange method (off-line). However, this method is labor intensive and not practical when rapid information is required for early identification of the metabolites. To further confirm the fragmentation assignment and to examine if the lower H/D exchange efficiency associated with in-ion-source exchange can cause any difficulties with

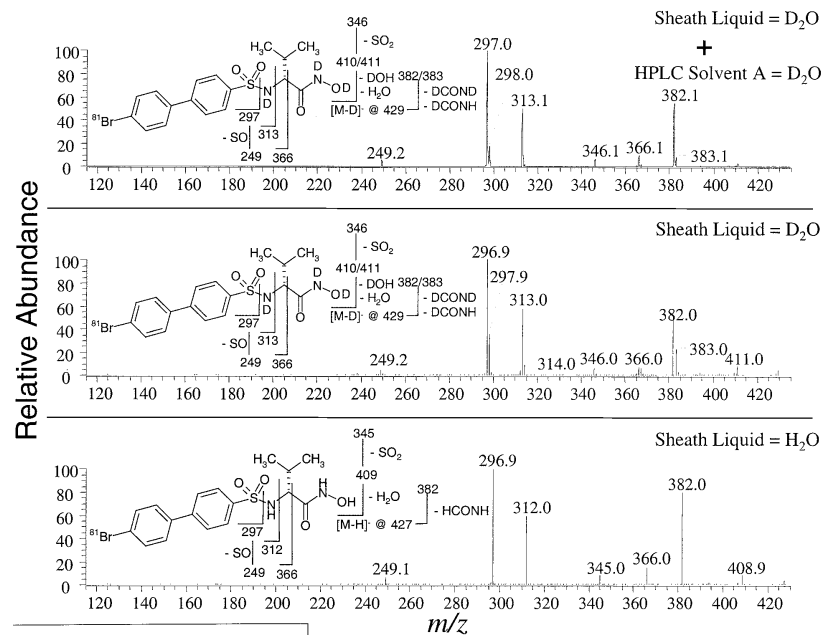

Figure 4. A comparison of LC-MS/MS spectra of PD 0200126 standard (MS isolation of deprotonated molecules that contained ${ }^{81} \mathrm{Br}$ ) obtained using off-line $\mathrm{H} / \mathrm{D}$ exchange followed by HPLC using deuteriated mobile phase and $\mathrm{D}_{2} \mathrm{O}$ as the sheath liquid (top panel), on-line $\mathrm{H} / \mathrm{D}$ exchange using aqueous mobile phase and $\mathrm{D}_{2} \mathrm{O}$ as the sheath liquid (middle panel), and aqueous mobile phase and $\mathrm{H}_{2} \mathrm{O}$ as the sheath liquid (bottom panel).

product ion interpretation, deprotonated molecules that contained ${ }^{81} \mathrm{Br}$ that had undergone three exchanges (less one exchangeable proton due to ionization) were selected and subjected to tandem mass spectrometry. Upon CID, the precursor ion of $\mathrm{m} / \mathrm{z} 429$ formed fragment ions of 249, 297, and 312. Unlike the in-ion-source $\mathrm{H} / \mathrm{D}$ exchange product ion spectrum of ${ }^{79} \mathrm{Br}-\mathrm{PD}$ 0200126, the in-ion-source H/D exchange product ion spectrum of ${ }^{81} \mathrm{Br}$-PD 0200126 was less complex (Figure 4 middle panel) and matched very closely the product ion spectrum obtained following off-line solution phase H/D exchange (Figure 4 top panel). A 2 Da mass shift of the fragment ions following the H/D exchange confirmed the fragmentation assignment (Figures 3 and 4 middle and top panel).

To evaluate the sensitivity and demonstrate the validity of this approach at the early drug discovery stage, we tested the method using rabbit plasma extracts. As shown in Figure 5, rabbit plasma showed three prominent UV components associated with metabolites $\mathrm{M}_{7}, \mathrm{M}_{8}$ and $\mathrm{M}_{9}$, respectively, at $\mathrm{m} / \mathrm{z} 425,310$, and 410 (metabolites $\mathrm{M}_{1}-\mathrm{M}_{6}$ were characterized in a separate study involving rat and mouse hepatocytes). Trace amounts of PD 0200126 and $\mathrm{M}_{10}$ were also observed at $\mathrm{m} / \mathrm{z} 425$ and 409, respectively. Structure elucidation of these metabolites was achieved by the combined use of LC-MS and LC-MS/MS where $\mathrm{H}_{2} \mathrm{O}$ was used as both an aqueous component of the mobile phase and sheath liquid while conducting in-ion-source $\mathrm{H} / \mathrm{D}$ exchange experiments.

\section{Metabolite $M_{7}$}

Metabolite $\mathrm{M}_{7}$ which had a similar nominal mass as the parent drug (Figure 5), eluted at $36.1 \mathrm{~min}$. The odd 


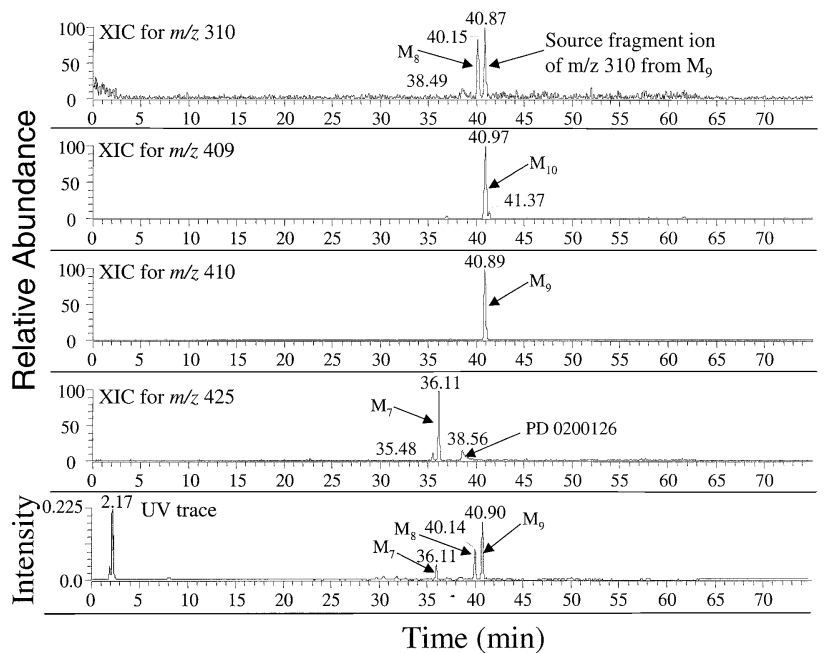

Figure 5. Extracted ion chromatograms for PD 0200126, $M_{7}, M_{8}$ $\mathrm{M}_{9}$, and $\mathrm{M}_{10}$ obtained using plasma following an oral administration of $300 \mathrm{mg} / \mathrm{kg}$ of PD 0200126 to rabbits.

value of $\mathrm{m} / \mathrm{z}$ for the metabolite $\mathrm{M}_{7}$ ion indicates that $\mathrm{M}_{7}$ bears an even number of nitrogen atoms. When $\mathrm{H}_{2} \mathrm{O}$ was used as the sheath liquid, $\mathrm{M}_{7}$ showed similar isotopic cluster pattern as the parent, however, when subjected to H/D exchange, the isotopic cluster pattern of $\mathrm{M}_{7}$ was different from that of the parent drug. As shown in Figure 2, when PD 0200126 was subjected to $\mathrm{H} / \mathrm{D}$ exchange, the most abundant isotopic cluster ion shifted by 2 Da whereas with $\mathrm{M}_{7}$ a 3 Da shift was observed (Figure 6). For metabolite $\mathrm{M}_{7}$, a 3 Da increase is in agreement with the presence of four exchangeable hydrogens in the proposed monohydroxy-PD 0200126amide or monohydroxy-PD 0307442 structure. Under the LC-MS/MS conditions used, $\mathrm{M}_{7}$ showed similar fragmentation pattern as the parent except with different relative abundance (Figure 7 bottom panel). The

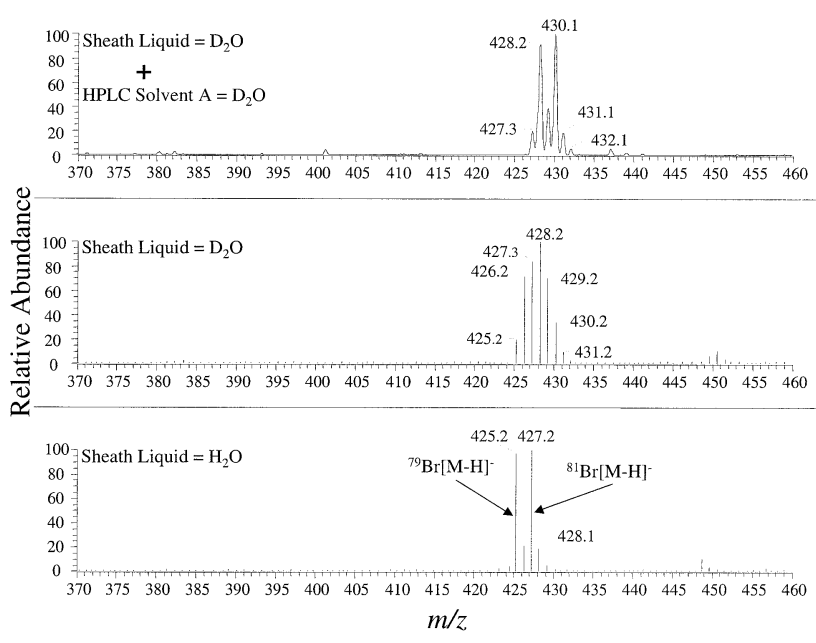

Figure 6. A comparison of LC-MS spectra of $\mathrm{M}_{7}$ metabolite obtained using off-line H/D exchange followed by HPLC using deuteriated mobile phase and $\mathrm{D}_{2} \mathrm{O}$ as the sheath liquid (top panel), on-line $\mathrm{H} / \mathrm{D}$ exchange using aqueous mobile phase and $\mathrm{D}_{2} \mathrm{O}$ as the sheath liquid (middle panel), and aqueous mobile phase and $\mathrm{H}_{2} \mathrm{O}$ as the sheath liquid (bottom panel).

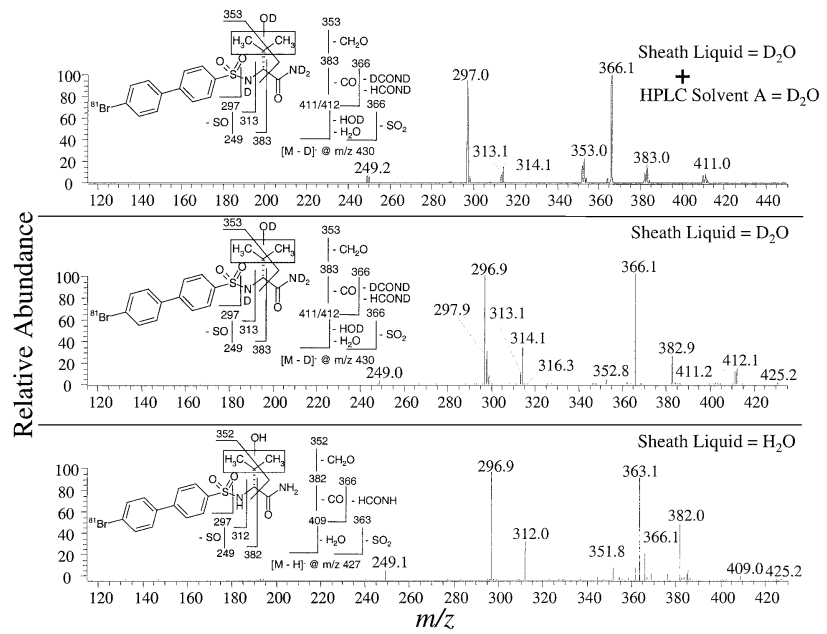

Figure 7. A comparison of LC-MS/MS spectra of metabolite $\mathrm{M}_{7}$ (MS isolation of deprotonated molecules that contained ${ }^{81} \mathrm{Br}$ ) obtained using off-line H/D exchange followed by HPLC using deuteriated mobile phase and $\mathrm{D}_{2} \mathrm{O}$ as the sheath liquid (top panel), on-line $\mathrm{H} / \mathrm{D}$ exchange using aqueous mobile phase and $\mathrm{D}_{2} \mathrm{O}$ as the sheath liquid (middle panel), and aqueous mobile phase and $\mathrm{H}_{2} \mathrm{O}$ as the sheath liquid (bottom panel).

major fragment ion at $m / z 297$ suggested that the bromo-biphenyl-4-sulfonyl moiety is intact. Fragment ion at $m / z 312$ suggested that the bromo-biphenyl-4sulfonylamino moiety is also intact and modification, possibly hydroxylation, is taking place on the 3-methylbutyramide moiety. Unlike the parent, metabolite $M_{7}$ underwent fragmentation to give abundant fragment ions corresponding to $\left[\mathrm{M}-\mathrm{H}-\mathrm{SO}_{2}\right]^{-}$at $\mathrm{m} / \mathrm{z}$ 363. Where a similar fragmentation behavior is observed with metabolite $\mathrm{M}_{10}$ (PD 0307442) or PD 0200126-amide (MS/MS spectrum not provided). Thus suggesting that this metabolite is possibly a monohydroxylated derivative of PD 0200126-amide. In addition to showing the fragment ions present in the MS/MS spectrum of the parent (Figure 7 bottom panel), $\mathrm{M}_{7}$ also gave trace amounts of fragment ion corresponding to $\left[\mathrm{M}-\mathrm{H}-\mathrm{H}_{2} \mathrm{O}\right]^{-}$at $m / z 409$. This fragment ion further supported the structural assignment provided for metabolite $M_{7}$.

Additionally, post H/D exchange MS/MS spectrum of the precursor ions of $\mathrm{m} / \mathrm{z} 430$ clearly supports the proposed structure for metabolite $M_{7}$ (Figure 7 top and middle panels). As shown in the fragmentation scheme, the major fragment ion at $\mathrm{m} / \mathrm{z} 297$ suggests that the bromo-biphenyl-4-sulfonyl moiety is intact. Fragment ions at $m / z 313 / 314$ suggest that the bromo-biphenyl-4sulfonylamino moiety is intact and confirms the presence of an exchangeable hydrogen. As expected, the fragment ion at $m / z 363$ corresponding to $\left[\mathrm{M}-\mathrm{H}-\mathrm{SO}_{2}\right]^{-}$ shifted by $3 \mathrm{Da}$ to $\mathrm{m} / \mathrm{z}$ 366. Both the H/D exchange information and the product-ion-spectra suggest that $\mathrm{M}_{7}$ is a metabolite where isopropyl moiety of PD 0307442 is modified by hydroxylation. As shown in the fragmentation scheme (Figure 7), observation of the fragment ion at $m / z 352$ and the associated deuterium exchanged fragment ion at $m / z 353$ suggests that hy- 
droxylation is most likely occurring on the methyl portion of the isopropyl moiety.

\section{Metabolite $M_{8}$}

Metabolite $\mathrm{M}_{8}$ eluted at $40.2 \mathrm{~min}$ (Figure 5) and gave deprotonated molecule that contained ${ }^{79} \mathrm{Br}$ and ${ }^{81} \mathrm{Br}$ respectively at $m / z 310$ and 312 . The even value of $\mathrm{m} / \mathrm{z}$ for the metabolite $\mathrm{M}_{8}$ ion indicates that $\mathrm{M}_{8}$ bears an odd number of nitrogen atoms. Under the MS/MS conditions used, precursor ions of $m / z 310$ fragmented to give ions of $\mathrm{m} / \mathrm{z} 246$ corresponding to [M-H-SO $]_{2}{ }^{-}$(MS/MS spectrum not shown). Similarly, precursor ions of $\mathrm{m} / \mathrm{z}$ 312 fragmented to give ions of $\mathrm{m} / \mathrm{z} 248$ corresponding to [M-H-SO $\left.]_{2}\right]^{-}$(MS/MS spectrum not shown). Loss of 64 Da suggested that sulfone is part of the metabolite and the fragment ion of $\mathrm{m} / \mathrm{z} 248$ suggested that bromobiphenylamide is intact. Following ESI source H/D exchange, the molecular ion cluster shifted from 310/ 312 to $311 / 313$ and confirmed the presence of an exchangeable hydrogen. Based on this information a structure of bromo-biphenylsulfonamide was proposed for metabolite $\mathrm{M}_{8}$ (Table 1). Finally, the proposed structure was confirmed by synthesis of reference standard (PD 0408208). The retention time, electrospray mass spectrum, and the tandem mass spectrum of the synthetic standard was in close agreement with that obtained from the metabolite $\mathrm{M}_{8}$ formed in vitro and in vivo.

\section{Metabolite $M_{9}$}

Metabolite $\mathrm{M}_{9}$ eluted at $40.9 \mathrm{~min}$ and gave deprotonated molecule that contained ${ }^{79} \mathrm{Br}$ and ${ }^{81} \mathrm{Br}$ respectively at $\mathrm{m} / \mathrm{z} 410$ and 412 . The even value of $\mathrm{m} / \mathrm{z}$ for the metabolite $\mathrm{M}_{9}$ ion indicates that $\mathrm{M}_{9}$ bears an odd number of nitrogen atoms. Precursor ions of $\mathrm{m} / \mathrm{z} 410$ fragmented to give ions of $m / z 247,295$, and 310 (Figure 8 bottom panel). The fragment ions of $\mathrm{m} / \mathrm{z} 295$ and 310 suggested that bromo-biphenylsulfonyl and bromo-biphenylsulfonylamino moieties are intact, respectively. Most likely, the fragment ion at $\mathrm{m} / \mathrm{z} 247$ is associated with $[295-\mathrm{SO}]^{-}$. Based on these MS/MS data and the molecular weight information, a structure of PD 0200126-carboxylic acid was proposed. To confirm the structural assignment, $\mathrm{H}_{2} \mathrm{O}$ and $\mathrm{D}_{2} \mathrm{O}$ were used separately as the sheath liquid and LC-MS and MS/MS data were collected. In addition to the molecular ion cluster at $m / z 410 / 412\left(\mathrm{H}_{2} \mathrm{O}\right.$ sheath) and $411 / 413\left(\mathrm{D}_{2} \mathrm{O}\right.$ sheath), $409 / 411\left(\mathrm{H}_{2} \mathrm{O}\right.$ sheath) and $410 / 412\left(\mathrm{D}_{2} \mathrm{O}\right.$ sheath) were also observed in the LC-MS spectra. The latter two pairs are most likely originating from combination of the following: Co-eluting metabolite $\mathrm{M}_{10}$, co-eluting metabolite undergoing a single deuterium exchange and some unexchanged $\mathrm{M}_{9}$. When $\mathrm{D}_{2} \mathrm{O}$ was used as the sheath liquid, the majority of the molecular ion cluster from $\mathrm{M}_{9}$ shifted by $1 \mathrm{Da}$ to $411 / 413$. Increment of a $\mathrm{Da}$ is in agreement with the proposed structure of PD 0200126carboxylic acid where two exchangeable hydrogens are

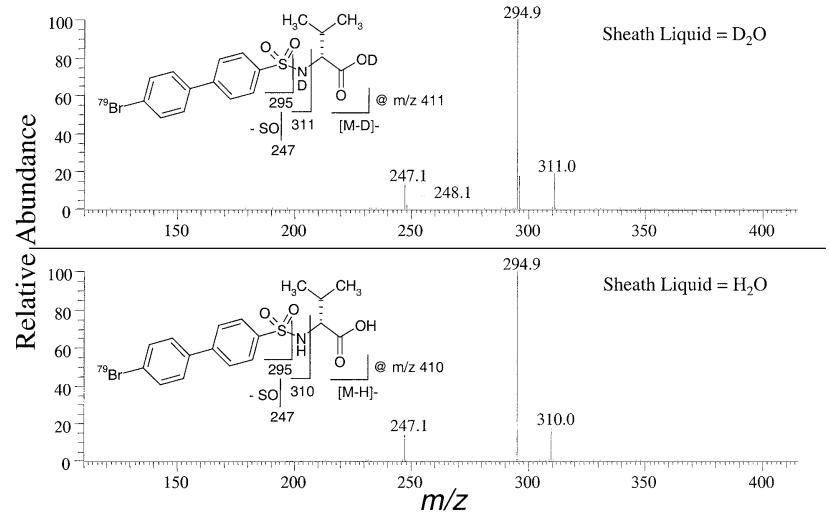

Figure 8. A comparison of LC-MS/MS spectra of metabolite $\mathrm{M}_{9}$ obtained using $\mathrm{H}_{2} \mathrm{O}$ (bottom panel) and $\mathrm{D}_{2} \mathrm{O}$ (top panel) as the sheath liquid.

present and one is removed for ionization. To further confirm the structural assignment, LC-MS/MS spectra were collected separately using $\mathrm{H}_{2} \mathrm{O}$ and $\mathrm{D}_{2} \mathrm{O}$ as the sheath liquid (Figure 8). Presence of ions of $\mathrm{m} / \mathrm{z} 247$ and 295 in both spectra plainly shows that bromo-biphenylsulfonyl moiety is intact. Mass shift of the fragment ion at $\mathrm{m} / \mathrm{z} 310$ to 311 clearly suggests the presence of an exchangeable hydrogen and the amide moiety of bromo-biphenylsulfonamide is intact. The retention time, electrospray mass spectrum, and the tandem mass spectrum of the synthetic standard (PD 0169469) was in close agreement with that obtained from the metabolite $\mathrm{M}_{9}$ formed in vitro and in vivo.

\section{Metabolite $M_{10}$}

Metabolite $\mathrm{M}_{10}$, a minor UV component in the rabbit plasma, eluted at $41.0 \mathrm{~min}$ and showed deprotonated molecule that contained ${ }^{79} \mathrm{Br}$ and ${ }^{81} \mathrm{Br}$ respectively at $\mathrm{m} / \mathrm{z} 409$ and 411 . The odd value of $\mathrm{m} / \mathrm{z}$ for the metabolite $\mathrm{M}_{10}$ ion indicates that $\mathrm{M}_{10}$ bears an even number of nitrogen atoms. Molecular weight of 410, corresponding to a decrease of $16 \mathrm{Da}$ over the molecular weight of PD 0200126 suggests that metabolite $\mathrm{M}_{10}$ is most likely an amide metabolite. Comparison of the product ion spectrum of $\mathrm{M}_{10}$ with that of PD 0200126 reference standard and presence of product ions of $\mathrm{m} / \mathrm{z} 295$ and 310 suggested that bromo-biphenylsulfonyl and bromobiphenylsulfonylamino moieties are intact, respectively (MS/MS spectrum not shown). Unlike the parent or the carboxylic acid metabolite, $\mathrm{M}_{10}$ undergoes fragmentation to give abundant ions corresponding to $[\mathrm{M}-\mathrm{H}-$ $\left.\mathrm{SO}_{2}\right]^{-}$at $m / z$ 345. To confirm the structural assignment, in-ion-source H/D exchange experiment was carried out on this metabolite. The molecular ion cluster was observed to shift by 2 Da to $411 / 413$ and indicated the presence of three exchangeable hydrogens. Upon CID, the precursor ion of $\mathrm{m} / \mathrm{z} 411$ gave fragment ions of $\mathrm{m} / \mathrm{z}$ 295, 311, and 347 and confirmed the structural assignment. Finally, the retention time, electrospray mass spectrum, and the tandem mass spectrum of the syn- 

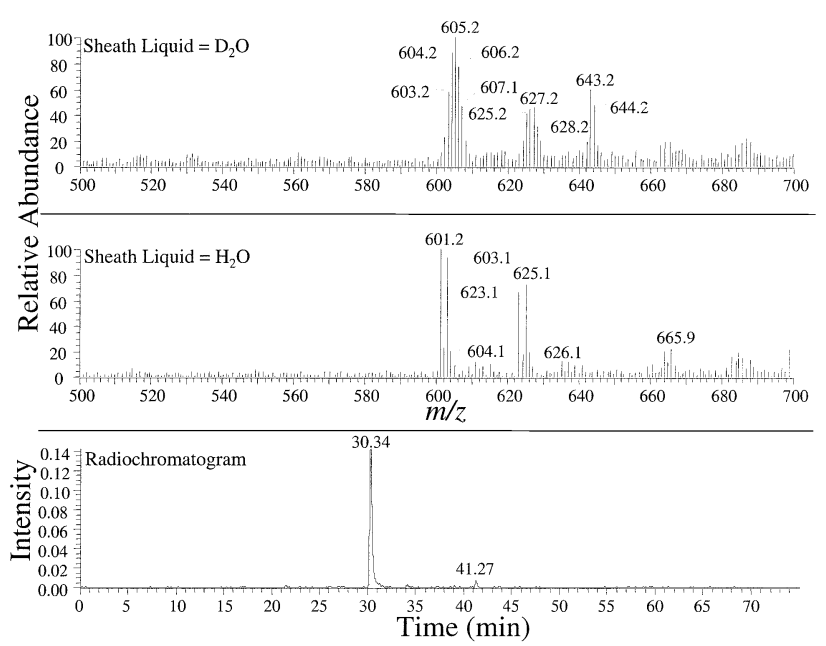

Figure 9. A comparison of LC-MS spectra of metabolite $\mathrm{M}_{2}$ obtained using $\mathrm{H}_{2} \mathrm{O}$ (middle panel) and $\mathrm{D}_{2} \mathrm{O}$ (top panel) as the sheath liquid and the corresponding radiochromatogram (bottom panel).

thetic standard (PD 0307442) were in close agreement with that obtained from the metabolite $\mathrm{M}_{10}$ formed in vitro and in vivo.

The next set of experiments were carried out to verify if the in-ion-source $H / D$ exchange is limited to Phase I modifications or modification by addition of 1-4 exchangeable hydrogens and is applicable to phase II modification such as glucuronidation which will result in addition of more than five exchangeable hydrogens. This set of experiments also demonstrated that the in-ion-source $\mathrm{H} / \mathrm{D}$ exchange is applicable not only to discovery (non-radiolabeled parent drug) metabolite identification but also to development studies (radiolabeled parent drug).

\section{Metabolite $\mathrm{M}_{2}$}

Following incubation of $\left[{ }^{14} \mathrm{C}\right] \mathrm{PD} 0200126$ with dog hepatocytes for $180 \mathrm{~min}$ (Figure 9), the major radioactive component representing more than $90 \%$ of the total radioactivity was associated with PD 0200126-glucuronide $\left(\mathrm{M}_{2}\right)$ and minor amounts of the radioactivity was associated with $M_{9}$. As shown in Figure 9, when subjected to in-ion-source $\mathrm{H} / \mathrm{D}$ exchange the molecular ion cluster observed at $\mathrm{m} / \mathrm{z} 601 / 603$ shifted to give ions at $m / z$ 602, 603, 604, 605, 606, 607, and 608. Similarly, [M $2 \mathrm{H}+\mathrm{Na}]^{-}$ions at $m / z 623 / 625$ shifted to $624,625,626$, 627,628 , and 629. Presence of ions of $\mathrm{m} / \mathrm{z} 606 / 608$ clearly show that all six exchangeable hydrogens (with one removed for forming the ion) in PD 0200126glucuronide are available for exchange and the precursors for these ions are most likely ions of $\mathrm{m} / \mathrm{z} 601 / 603$. Formation of the sodium adduct results in the removal of a second proton or deuterium and results in one less exchangeable hydrogen. These observations clearly show that $\mathrm{M}_{2}$ is a glucuronide conjugate of PD 0200126 and not a conjugate of $\mathrm{M}_{7}$. If $\mathrm{M}_{2}$ had been a glucuronide conjugate of $\mathrm{M}_{7}$, seven exchangeable hydrogens would be available for the exchange reaction. The proposed structure of $\mathrm{M}_{2}$ was further confirmed by $\beta$-glucuronidase hydrolysis experiments [28]. Structure elucidation of $\mathrm{M}_{2}$ further confirmed that the in-ion-source $\mathrm{H} / \mathrm{D}$ exchange is not limited to phase I type biotransformation where the metabolic addition of exchangeable hydrogens is limited and the experimental set-up is not limited to discovery type metabolite identification studies and it will allow one to obtain structural information as well as radioactivity quantitative information (using the split-flow technique) about the metabolites.

\section{Conclusions}

The use of mass spectrometer equipped with an ESI source and deuterium oxide $\left(\mathrm{D}_{2} \mathrm{O}\right)$ as the sheath liquid for performing on-line $\mathrm{H} / \mathrm{D}$ exchange experiments has been demonstrated. Experiments show that when using in-ion-source $\mathrm{H} / \mathrm{D}$ exchange the exchange efficiencies are not high as samples dissolved in $\mathrm{D}_{2} \mathrm{O}$ and the use of $\mathrm{D}_{2} \mathrm{O}$ mobile phase, the method described in this paper can be used to rapidly identify the number of exchangeable hydrogens of drugs and metabolites and to characterize them.

$\mathrm{H} / \mathrm{D}$ exchange experiments allowed us to distinguish the parent drug from metabolite $\mathrm{M}_{7}$, which had the same nominal mass and identical elemental composition. In addition, presence of the bromine atom, specifically the unique isotopic cluster distribution, in the parent drug allowed us to assess the effectiveness of the in-ion-source $\mathrm{H} / \mathrm{D}$ exchange for obtaining tandem mass spectrometry information. Comparison of the production-spectra obtained using the precursor ions formed following in-ion-source $\mathrm{H} / \mathrm{D}$ exchange and solution (off-line) H/D exchange with deuteriated mobile phase shows that structure elucidation of metabolites can be achieved by using precursor ions where the H/D exchange has not been driven to completion. Our experiments also show that in-ion-source H/D exchange is not limited to metabolites formed by addition of smaller number of exchangeable hydrogens such as the ones associated with Phase I biotransformation but also to Phase II biotransformation which usually results in metabolites formed by addition of five or more exchangeable hydrogens (glucuronidation and glutathione but not sulfation). Thus, by using in-ion-source $\mathrm{H} / \mathrm{D}$ exchange, some of the unambiguous metabolite identification, which can be very useful in the early stages of drug discovery, can be achieved without the use of large scale preparation for NMR or LC-NMR or performing solvent (off-line) H/D exchange involving the use of deuteriated mobile phase.

\section{Acknowledgments}

The authors would like to thank Kevin Conlon for his helpful suggestions and Jonathon Bauman, Jasminder Sahi, Erick Kindt, and Adriaan Cleton for providing some of the samples used in this study. 


\section{References}

1. Siddiqui, M. A. Improving Drug R\&D Efficiency. Chem. Eng. News 2001, (March 26), 275.

2. Czarnik, A. Combinatorial Chemistry. Anal. Chem. 1998, 70, 378A-386A.

3. Bertrand, M.; Jackson, P.; Walther, B. Rapid Assessment of Drug Metabolism in the Drug Discovery Process. Europ. J. Pharma. Sci. 2000, 11, S61-S72.

4. Zhang, H.; Henion, J.; Yang, Y.; Spooner, N. Application of Atmospheric Pressure Ionization Time-of-Flight Mass Spectrometry Coupled with Liquid Chromatography for the Characterization of in vitro Drug Metabolites. Anal. Chem. 2000, 72, 3342-3348.

5. Cox, K. A.; Clarke, N. J.; Rindgen, D.; Korfmacher, W. A. Higher Throughput Metabolite Identification in Drug Discovery: Current Capabilities and Future Trends. Am. Pharma. Rev. 2001, 4, 45-52.

6. Englander, S. W.; Sosnick, T. R.; Englander, J. J.; Mayne, L. Mechanisms and Uses of Hydrogen Exchange. Curr. Opin. Struct. Biol. 1996, 6, 18-23.

7. Bai, Y.; Sosnick, T. R.; Mayne, L.; Englander, S. W. Protein Folding Intermediates: Native-State Hydrogen Exchange. Science 1995, 269, 192-197.

8. Miranker, A.; Robinson, C. V.; Radford, S. E.; Aplin, R. T.; Dobson, C. M. Detection of Transient Protein Folding Populations by Mass Spectrometry. Science 1993, 262, 896-899.

9. Hunt, D. F.; McEwen, C. N.; Upham, R. A. Chemical Ionization Mass Spectrometry II: Differention of Primary, Secondary, and Tertiary Amines. Tetrahedron Lett. 1971, 28, 4539.

10. Hunt, D. F.; Sethi, S. K. Gas-Phase Ion/Molecule IsotopeExchange Reactions: Methodology for Counting Hydrogen Atoms in Specific Organic Structural Environments by Chemical Ionization Mass Spectrometry. J. Am. Chem. Soc. 1980, 102, 6953-6963.

11. Ranasinghe, A.; Cooks, R. G.; Sethi, S. K. Selective Isotopic Exchange of Polyfunctional Ions in Tandem Mass Spectrometry: Methodology, Applications, and Mechanism. Org. Mass Spectrom. 1992, 27, 77-88.

12. Gard, E.; Green, M. K.; Bregar, J.; Lebrilla, C. B. Gas-Phase Hydrogen/Deuterium Exchange as a Molecular Probe for the Interaction of Methanol and Protonated Peptides. J. Am. Soc. Mass Spectrom. 1994, 5, 623-631.

13. McLean, T.; New, A. P.; Haskins, N. J.; Camilleri, P. Deuteriated Solvents in High Performance Liquid ChromatographyFast Atom Bombardment Mass Spectrometry. J. Chem. Soc. Chem. Commun. 1992, 15, 1773-1775.

14. Edlund, P. O. Identification of Amperozide Metabolites in Urine from Rats, Rabbits, Dogs, and Man by Frit-FAB LC/MS Using Deuterated Solvents to Gain Additional Structural Information. J. Mass Spectrom. 1995, 30, 1380-1392.

15. Siegel, M. M. Hydrogen-Deuterium Exchange Studies Utilizing a Thermospray Mass Spectrometer Interface. Anal. Chem. 1988, 60, 2090-2095.

16. Edmonds, C. G.; Pomerantz, S. C.; Hsu, F. F.; McCloskey, J. A. Thermospray Liquid Chromatography/Mass Spectrometry in Deuterium Oxide. Anal. Chem. 1988, 60, 2314-2317.
17. Katta, V.; Chait, B. T. Conformational Changes in Proteins Probed by Hydrogen-Exchange Electrospray-Ionization Mass Spectrometry. Rapid Commun. Mass Spectrom. 1991, 5, 214-217.

18. Katta, V.; Chait, B. T. Hydrogen/Deuterium Exchange Electrospray Ionization Mass Spectrometry: A Method for Probing Protein Conformational Changes in Solution. J. Am. Chem. Soc. 1993, 115, 5(14), 6317-6321.

19. Ramanathan, R.; Gross, M. L.; Zielinski, W. L.; Layloff, T. P. Monitoring Recombinant Protein Drugs: A Study of Insulin by H/D Exchange and Electrospray Ionization Mass Spectrometry. Anal. Chem. 1997, 69, 5142-5145.

20. Robinson, C. V.; Gross, M.; Eyles, S. J.; Ewbank, J. J.; Mayhew, M.; Hartl, F. U.; Dobson, C. M.; Radford, S. E. Conformational of GroEL-Bound $\alpha$-Lactalbumin Probed by Mass Spectrometry. Nature 1994, 372, 646-651.

21. Wagner, D. S.; Anderegg, R. J. Conformation of Cytochrome c Studied by Deuterium Exchange-Electrospray Ionization Mass Spectrometry. Anal. Chem. 1994, 66, 706-711.

22. Rosenbaum, D. M.; Roy, S.; Hecht, M. H. Screening Combinatorial Libraries of de novo Proteins by Hydrogen-Deuterium Exchange and Electrospray Mass Spectrometry. J. Am. Chem. Soc. 1999, 121, 9509-9513.

23. Winger, B. E.; Light-Wahl, K. J.; Rockwood, A. L.; Smith, R. D. Probing Qualitative Conformation Differences of Multiply Protonated Gas-Phase Proteins via H/D Isotopic Exchange with $\mathrm{D}_{2}$ O. J. Am. Chem. Soc. 1992, 114, 5897-5898.

24. Hemling, M. E.; Conboy, J. J.; Bean, M. F.; Mentzer, M.; Carr, S. A. Gas Phase Hydrogen/Deuterium Exchange in Electrospray Ionization Mass Spectrometry as a Practical Tool for Structure Elucidation. J. Am. Soc. Mass Spectrom. 1994, 5, $434-442$.

25. Liu, D. Q.; Hop, C. E. C. A.; Beconi, M. G.; Mao, A.; Chiu, S.-H. L. Use of On-Line Hydrogen/Deuterium Exchange to Facilitate Metabolite Identification. Rapid Commun. Mass Spectrom. 2001, 15, 1832-1839.

26. Ohashi, N.; Furuuchi, S.; Yoshikawa, M. Usefulness of the Hydrogen-Deuterium Exchange Method in the Study of Drug Metabolism Using Liquid Chromatography-Tandem Mass Spectrometry. J. Pharm. Biomed. Anal. 1998, 18, 325-334.

27. O'Brien, P. M.; Ortwine, D. F.; Pavlovsky, A. G.; Picard, J. A.; Sliskovic, D. R.; Roth, B. D.; Dyer, R. D.; Johnson, L. L.; Man, C. F.; Hallak, H. Structure-Activity Relationships and Pharmacokinetic Analysis for a Series of Potent, Systemically Available Biphenylsulfonamide Matrix Metalloproteinase Inhibitors. J. Med. Chem. 2000, 43, 156-166.

28. Sahi, J.; Bauman, J.; Ramanathan, R.; Cleaton, A. Unpublished.

29. Palmer, M. E.; Tetler, L. W.; Wilson, I. D. Hydrogen/Deuterium Exchange Using a Coaxial Sheath-Flow Interface for Capillary Electrophoresis/Mass Spectrometry. Rapid Commun. Mass Spectrom. 2000, 14, 808-817.

30. McCloskey, J. A. Introduction of Deuterium by Exchange for Measurement by Mass Spectrometry Mass Spectrometry, Vol. CXCIII. Academic Press: San Diego, 1990; pp 329-338.

31. Verma, S.; Pomerantz, S. C.; Sethi, S. K.; McCloskey, J. A. Fast Atom Bombardment Mass Spectrometry Following Hydrogen-Deuterium Exchange. Anal. Chem. 1986, 58, 2898-2902. 